\title{
Aortic intimal intussusception during acute type B aortic dissection endovascular repair
}

\author{
Zhi-Yuan Wu ${ }^{1,2}$, Peng $\mathrm{Li}^{1,2}$, Ji-Yang Wang ${ }^{1,2}$, Yong-Peng Diao ${ }^{1,2}$, Zuo-Guan Chen ${ }^{1,2}$, Yu-Qing Miao, ${ }^{1,2}$, \\ Zhi-Gang Chang ${ }^{3}$, Hong Zhang ${ }^{4}$, Yong-Jun $\mathrm{Li}^{1,2}$ \\ ${ }^{1}$ Department of Vascular Surgery, Beijing Hospital, National Center of Gerontology, Beijing 100730, China; ${ }^{2}$ Peking Union Medical College \\ and Chinese Academy of Medical Sciences, Beijing 100730, China; ${ }^{3}$ Department of Critical Care Medicine, Beijing Hospital, National Center of \\ Gerontology, Beijing 100730, China; ${ }^{4}$ Department of Vascular Surgery, Affiliated Hospital of Chengde Medical University, Chengde 067000, China \\ Correspondence to: Yongjun Li, PhD, MD. Department of Vascular Surgery, Beijing Hospital, No. 1, Dahua Road, Dongdan, Dongchen District, \\ Beijing 100730, China. Email: liyongjun4679@bjhmoh.cn.
}

\begin{abstract}
Aortic intimal intussusception (AoII) is rare, especially during the endovascular repair of acute uncomplicated type B aortic dissection. Here we present a case of 47-year-old man who suffered AoII during the endovascular repair of type B aortic dissection. An abdominal aortic stent was inserted to recanalize the aorta, but failed. He was immediately transferred to our department from the local hospital. Computed tomography angiography confirmed the AoII and showed thrombus in the abdominal aortic stent. Hybrid operation was performed. Final angiography showed patency of the aorta. His postoperative period was uneventful and was discharged on the postoperative $8^{\text {th }}$ day. No complications happened during the $6^{\text {th }}$ month follow-up.
\end{abstract}

Keywords: Aortic intimal intussusception (AoII); endovascular repair; aortic dissection; complication

Submitted Jul 05, 2019. Accepted for publication Sep 23, 2019.

doi: $10.21037 /$ atm.2019.10.52

View this article at: http://dx.doi.org/10.21037/atm.2019.10.52

\section{Introduction}

Since the first autopsy reported in 1887 (1), more than 80 cases of aortic intimal intussusception (AoII) have been reported in English-written literatures. AoII is a rare and potential life-threatening disease (2). It is usually secondary to aortic dissection and is developed by the detached intima from its origin (3). Once formed, it can cause malperfusion, posing a great threat to life. However, it is seldom reported during the thoracic endovascular repair (TEVAR) of uncomplicated type B aortic dissection (3). Here we report a case of AoII with malperfusion to raise the awareness of AoII during endovascular repair of aortic dissection.

\section{Case presentation}

A 47-year-old man was diagnosed with acute type B aortic dissection in local hospital, complaining of chest and back pain for 4 days (Figure 1). He smoked for 20 years, without other medical history. He was performed of TEVAR in local hospital. After releasing the thoracic aortic stent, no signs of blood flow was observed in the abdominal aortic branches distal to the celiac trunk. Another aortic stent was inserted to recanalize the abdominal aorta, which was occluded by AoII, but finally failed.

The patient was immediately transferred to our hospital, complaining of cold in bilateral lower extremities and back pain, but of no chest or abdominal pain. Physical examination revealed no pulse in bilateral femoral and femoral beyond arteries, along with subnormal skin temperature in bilateral lower extremities. The blood pressure was $190 / 110 \mathrm{mmHg}$. The heart rate was 100 per minute and the $\mathrm{SPO}_{2} \%$ was $93 \%$. Imaging examination confirmed the AoII and also showed thrombus in the abdominal aortic stent (Figure 2A). Occlusions or stenosis happened in the celiac trunk ostium, superior mesenteric artery (Figure $2 B$ ) and iliac arteries. Bilateral renal arteries were supplied by the false lumen (Figure $2 C, D$ ). The levels of alanine transaminase (ALT), aspartate transaminase 


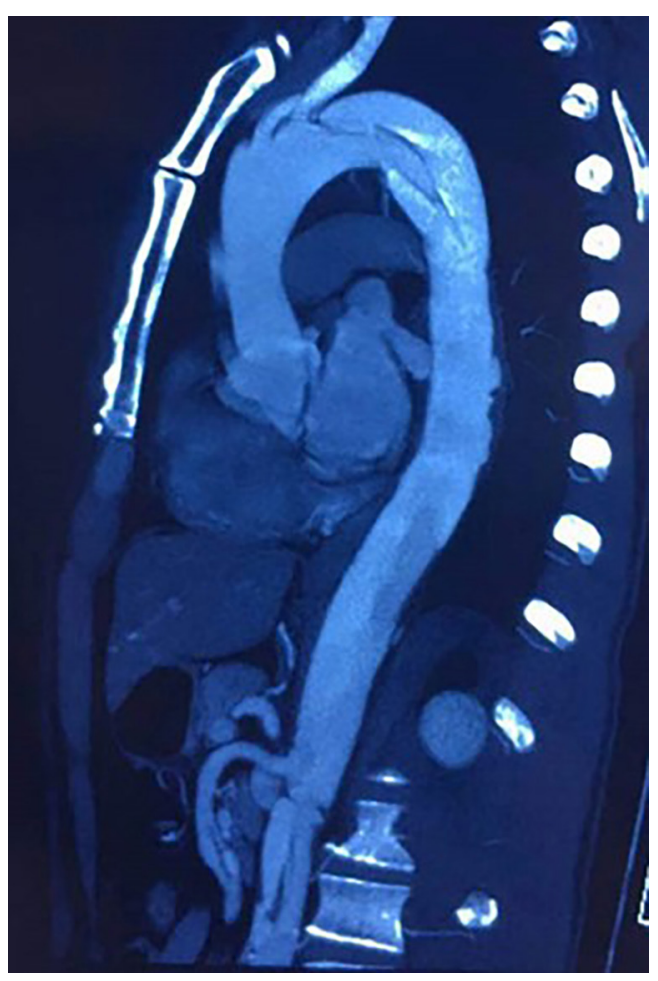

Figure 1 Acute type B aortic dissection.

(AST), creatinine kinase $(\mathrm{CK})$ and creatinine were separately $370 \mathrm{U} / \mathrm{L}, 648 \mathrm{U} / \mathrm{L}, 1,294 \mathrm{U} / \mathrm{L}$ and $107 \mathrm{mmol} / \mathrm{L}$. AoII and malperfusion were then diagnosed.

With the patient's and his family's consent, hybrid surgeries were applied after multidisciplinary discussion. Open surgery was performed to remove the intussuscepted aortic intima. When the abdominal cavity was exposed, the color of liver showed negative and the intestine peristalsis was inactive, as well as the pulse of mesenteric arteries. Abdominal aorta endarterectomy was applied to take out the failed abdominal aortic stent and to reconstruct the abdominal aorta and its branches (Figure 3). After aorta reconstruction, the intestine returned viable and the mesenteric arteries pulsated again. Endovascular repair was then performed in bilateral iliac arteries and stents were placed. Final angiography showed patency and no leakage of the aorta. Finally, bilateral femoral arteries and dorsal arteries pulsated again. During the operation, no transfusion was needed.

After operation, the patient was transferred to the intensive care unit, where he received mechanical ventilation and controlled blood pressure for 2 days. His postoperative period was uneventful. The creatinine decreased from $107 \mathrm{mmol} / \mathrm{L}$ at admission to $65 \mathrm{mmol} / \mathrm{L}$ at discharge. The computed tomography angiography (CTA) of the $8^{\text {th }}$ postoperative day showed patent in the aorta and no complications happened (Figure 4). He was discharged on the $8^{\text {th }}$ postoperative day. And blood pressure was monitored at home with routine antihypertensive drugs. No complications happened during the $6^{\text {th }}$ month follow-up (Figure 5).

\section{Discussion}

TEVAR has been the main current in dealing with the acute uncomplicated type B dissection. TEVAR-related mortality risk should be assessed before the operation (4). Many TEVAR-related complications are already known among vascular surgeons, such as spinal cord ischemia, etc. But TEVAR-related AoII is seldom reported (3). AoII is usually secondary to aortic dissection. It is mostly reported in type A aortic dissection, with few (1) in type B aortic dissection. Similar to other TEVAR-related AoII cases $(3,5,6)$, this patient also suffered AoII during the endovascular repair of uncomplicated type B aortic dissection. His intima may be torn by a strong radial force after the releasing of thoracic aortic stent, resulting in occlusion of abdominal aorta and no signs of blood flow.

AoII manifests differently based on the locations and the intussusception degree. For the AoII that locates in ascending aorta, the detached intima may fold back into the left ventricular outflow tract (7), leading to aortic regurgitation and the subsequent complications. It may also cause occlusions in the three main branches of arch, which may lead to stroke. While for those in type B aortic dissection, malperfusion may happen if the intima is intussuscepted to a large extent.

AoII can be detected by different imaging methods, such as computed tomography, echocardiogram and magnetic resonance (1). In this case, AoII was detected during the operation by digital subtraction angiography. When the patient was transferred to our hospital, another CTA was performed and further confirmed the lesion.

Methods to deal with the TEVAR-related AoII in descending aorta include immediate conversion to open surgery, percutaneous balloon fenestration and aspiration thrombectomy, etc. In this case, though local doctors tried to recanalize the abdominal aorta by fenestration with another aortic stent, the patient's situation didn't get better. Advanced aortic endovascular repair techniques may be helpful, such as parallel grafts. However, within the advanced endovascular techniques, it is difficult to establish a pathway between the aorta and the aortic stent. Besides, several parallel stents 

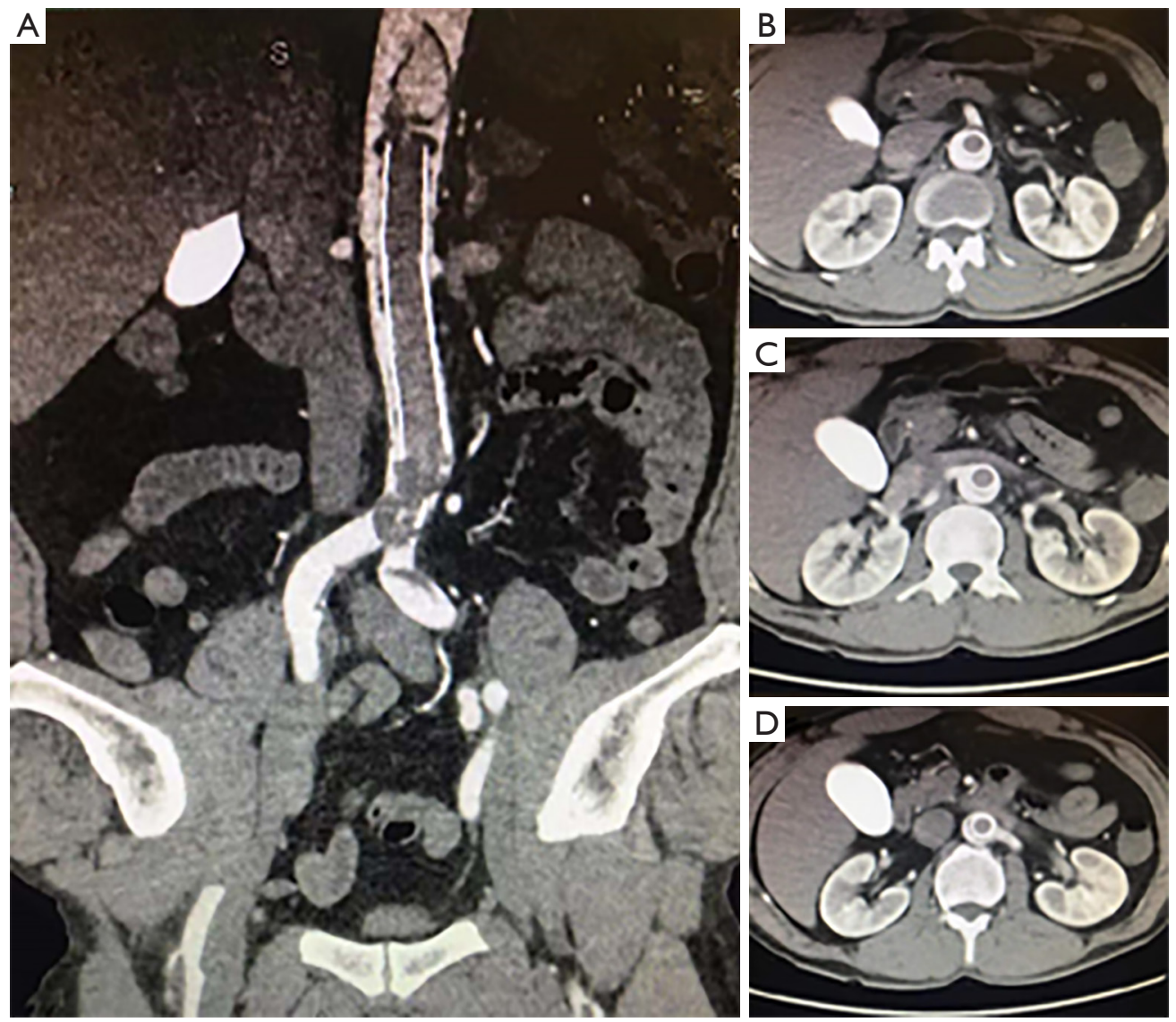

Figure 2 Computed tomography angiography (CTA) at admission. (A) The aortic intimal intussusception was confirmed and the thrombus can be seen in the abdominal aortic stent; (B) occlusions or stenosis happened in the superior mesenteric artery; (C,D) bilateral renal arteries were supplied by the false lumen.

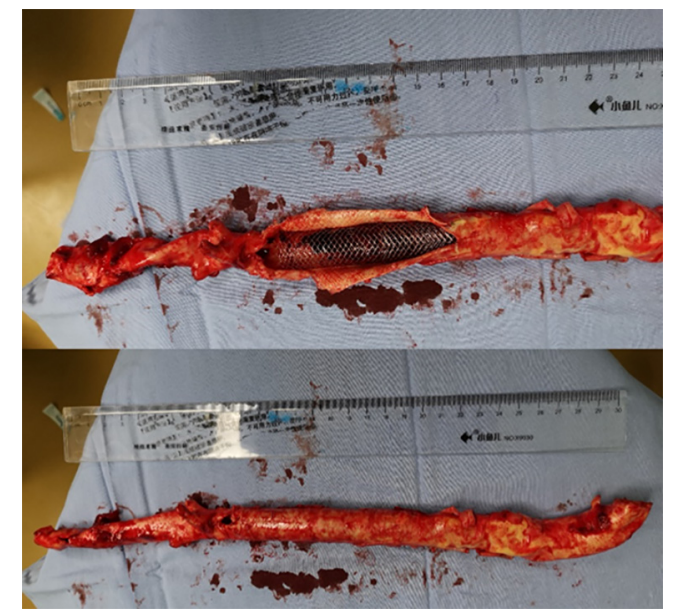

Figure 3 Removed intima and abdominal aortic stent. Thrombus can be seen in the stent. are needed to reconstruct the four visceral arteries, which may lead to dissatisfied patency. Another challenge is that this patient suffered abdominal malperfusion, which needed to be dealt with as soon as possible. Considering these factors, we decided to reconstruct the abdominal aorta and the branches by open surgeries. To minimize the wound, we adopted endovascular repair in bilateral iliac arteries. Though the results of $6^{\text {th }}$ month follow-up showed good, with no aorta rupture and no death, long-term follow up is still required.

\section{Conclusions}

AoII is rare, especially during the endovascular aortic repair of acute uncomplicated type B aortic dissection. Awareness of AoII should be raised and aorta endarterectomy in time can be life-saving. 

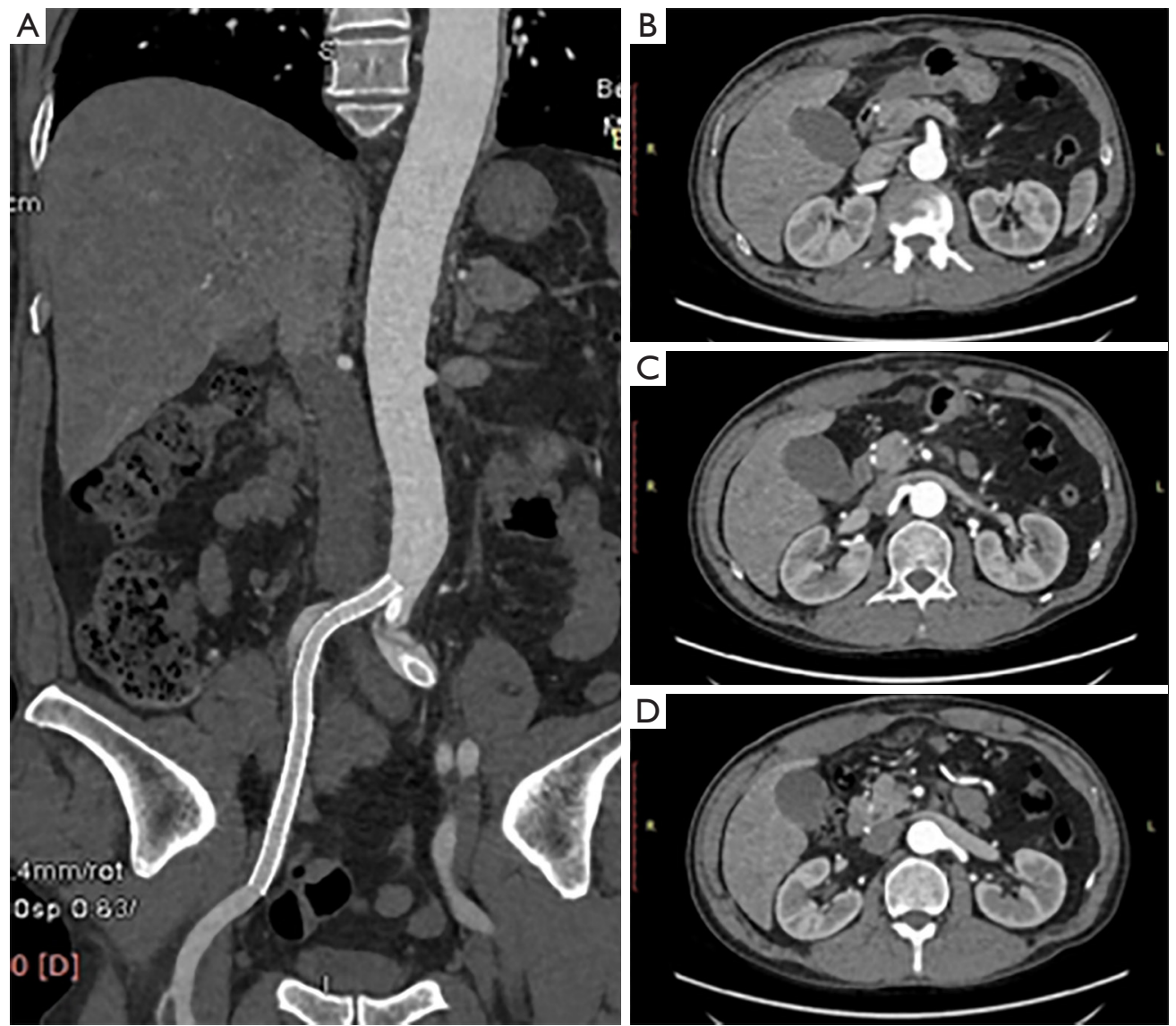

Figure 4 CTA results on the $8^{\text {th }}$ postoperative day. (A) CTA results on the $8^{\text {th }}$ postoperative day showed patent in the patient's aorta; (B,C,D) the superior mesenteric artery and bilateral renal arteries were supplied well without any occlusions or stenosis. CTA, computed tomography angiography.
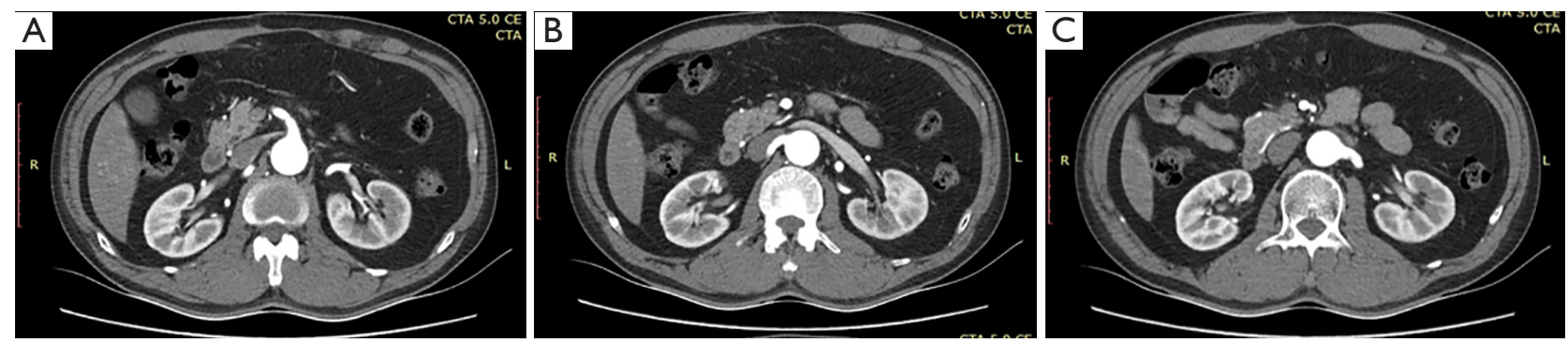

Figure 5 CTA results during the $6^{\text {th }}$ month follow-up. (A,B,C) No occlusion, stenosis and other complications happened to the abdominal aorta, superior mesenteric artery and bilateral renal arteries. CTA, computed tomography angiography.

\section{Acknowledgments}

Funding: Prof. Dr. YJ Li is funded by National Natural Science Foundation of China (No. 81870351), CAMS Innovation Fund for Medical Sciences (CIFMS, 2018-I2M-1-
002) and Beijing Hospital Clinical Research 121 Project (No. BJ-2018-089). Dr. YP Diao is supported by the Fundamental Research Funds for the Central Universities (No. 3332018174) and by Beijing Natural Science Foundation (No. 7192186). 


\section{Footnote}

Conflicts of Interest: The authors have no conflicts of interest to declare.

Ethical Statement: The authors are accountable for all aspects of the work in ensuring that questions related to the accuracy or integrity of any part of the work are appropriately investigated and resolved. Written informed consent was obtained from the patient and his family.

\section{References}

1. Roberts WC, Kapoor P, Main ML, et al. Acute Aortic Dissection With Intussusception of the Partition Between the True and False Channels Leading to Near Total Aortic Occlusion (True Aortic Stenosis). Am J Cardiol 2017;119:340-4.

2. Lajevardi SS, Sian K, Ward M, et al. Circumferential intimal tear in type A aortic dissection with intimo-intimal intussusception into left ventricle and left main coronary

Cite this article as: Wu ZY, Li P, Wang JY, Diao YP, Chen ZG, Miao YQ, Chang ZG, Zhang H, Li YJ. Aortic intimal intussusception during acute type $\mathrm{B}$ aortic dissection endovascular repair. Ann Transl Med 2019;7(22):700. doi: 10.21037/atm.2019.10.52 artery occlusion. J Thorac Cardiovasc Surg 2012;144:e21-3.

3. Havelka GE, Tomita TM, Malaisrie SC, et al. Two Cases of Aortic Intimal Intussusception During Endovascular Repair of an Acute Type B Dissection. J Endovasc Ther 2016;23:521-8.

4. Kilic A, Sultan IS, Arnaoutakis GJ, et al. Assessment of Thoracic Endografting Operative Mortality Risk Score: Development and Validation in 2,000 Patients. Ann Thorac Surg 2015;100:860-7.

5. Maynar M, Rostagno R, Zander T, et al. Intimal dehiscence in the abdominal aorta following balloon fenestration for type B dissection. J Endovasc Ther 2005;12:103-9.

6. Mirick AL, Patel HJ, Deeb GM, et al. Aortic intussusception complicating diagnostic angiography: recognition and management. Ann Thorac Surg 2013;95:1776-8.

7. Yanase Y, Ohkawa A, Inoue S, et al. Stanford Type A Acute Aortic Dissection with Intimal Intussusception. Ann Thorac Cardiovasc Surg 2018. [Epub ahead of print]. 